Conclusions: Self-reported adherence was high in our cohort with differences between the two groups. "Forgetfulness" regarding medication and keeping track of hospital appointments were the commonest reasons for non-adherence rather than deliberate non-adherence. Therefore novel behavioural or electronic cues for medication, including mobile app use, and appointment alerts could lead to improvement. Further work is required to identify whether a different personalised approach in the lupus and vasculitis patients can improve adhrence.

REFERENCE:

[1] Chambers SA, et al. Rheumatology. 2008;48: 266-271.

Disclosure of Interest: None declared

DOI: 10.1136/annrheumdis-2018-eular.5932

\section{AB1243 GETTING A HEEADSSS IN PSYCHOSOCIAL SCREENING: USE OF STANDARDISED CLINIC NOTE TEMPLATES FOR PSYCHOSOCIAL SCREENING IN A PAEDIATRIC RHEUMATOLOGY CLINIC}

E. Brennan Treemarcki, J. Szymonifka, A. Adams, N. Pan, S. Taber, K. Onel. Hospital for Special Surgery, New York, USA

Background: Children and adolescents with rheumatic diseases are at risk for psychosocial challenges related to illness, treatment, and normal development and should receive psychosocial screening. Psychosocial assessments have been successfully utilised in other paediatric specialties, including oncology and gastroenterology. A common psychosocial screening tool is the HEEADSSS (home environment, education/employment, eating, activities, drugs, sexuality, suicide/depression, and safety). This tool is easy to remember, quickly administered, and modifiable for younger children.

Objectives: We conducted a quality improvement project aimed to increase psychosocial screening by providers in a single paediatric rheumatology clinic by instituting standardised follow up templates for juvenile idiopathic arthritis (JIA) and systemic lupus erythematosus (SLE) including the HEEADSSS assessment. Methods: The quality improvement intervention evaluated in this study involved the creation of disease specific follow up note templates inclusive of the HEEADSSS assessment and introduction of the templates to the paediatric rheumatology fellows clinic. Charts of patients with an ICD-10 diagnosis of JIA or SLE were reviewed during a 3 month window prior to (February-April 2017) and after (October-December 2017) the intervention. Charts were reviewed for presence of 2 elements of the HEEADSSS assessment documented within the previous 12 months: smoking exposure (all patients) and sexual activity (ages 11 years and up). The proportion of patients with completed HEEADSSS assessment components pre- and post-intervention were compared using Fisher's exact test.

Results: Chart review included 36 patients pre- and 42 post-intervention (table 1). There was an increase in assessment of both measures in the period immediately following the intervention. Assessment of smoking exposure increased from $0.0 \%$ to $21.4 \%(p=0.003)$ with greater but not significant improvement in SLE versus JIA patients $(29.4 \%$ versus $16.4 \%, p=0.45)$. Assessment of sexual activity in patients 11 years and older increased from $13.0 \%$ to $37.5 \%(p=0.09)$, although this comparison may be underpowered due to decreased sample sizes.

Abstract AB1243 - Table 1. Documentation status pre- and post- implementation of standardised follow up templates for providers.

\begin{tabular}{lccc}
\hline & Pre-Intervention & Post-Intervention & p-value \\
\hline Smoking assessed & & & \\
Overall & $0 / 36(0.0 \%)$ & $9 / 42(21.4 \%)$ & 0.003 \\
JIA & $0 / 20(0.0 \%)$ & $4 / 25(16.0 \%)$ & 0.12 \\
SLE & $0 / 16(0.0 \%)$ & $5 / 17(29.4 \%)$ & 0.04 \\
Sexual Activity (11+) assessed & & & \\
Overall & $3 / 23(13.0 \%)$ & $9 / 24(37.5 \%)$ & 0.09 \\
JIA & $1 / 9(11.1 \%)$ & $2 / 8(25.0 \%)$ & 1.00 \\
SLE & $2 / 14(14.3 \%)$ & $7 / 16(43.8 \%)$ & 0.14 \\
\hline
\end{tabular}

Conclusions: Introduction of standardised note templates with the HEEADSSS assessment has resulted in preliminary improvement in psychosocial screening by providers in a single paediatric rheumatology clinic as demonstrated by improved rates of screening for smoking exposure and a trend towards improved screening rates for sexual activity. Additional studies are needed to confirm these findings, and future studies will assess whether the improvement seen is sus tained and whether usage of this template can be expanded to other paediatric patient populations

Acknowledgements: We thank the EMR team for helping develop the note templates and the fellows for adopting these templates.

Disclosure of Interest: None declared

DOI: 10.1136/annrheumdis-2018-eular.7395

\section{AB1244 QUALITY LIFE IN PATIENTS WITH RHEUMATIC DISEASE, NON-RHEUMATIC DISEASES AND HEALTHY POPULATION}

F.M. Cuervo ${ }^{1}$, on behalf of Grupo de investigación Espondiloartropatias,

Universidad de La Sabana-Hospital Militar Central, A.M. Santos ${ }^{1}$, J.C. Rueda ${ }^{1}$,

I. Angarita ${ }^{1}$, E.L. Saldarriaga, R. Giraldo ${ }^{1}$, J. Ballesteros ${ }^{1}$, I. Pelaez ${ }^{2}$, E. Forero ${ }^{3}$,

J. Ramirez ${ }^{4}$, C. Toro ${ }^{4}$, J. Londono ${ }^{1} .{ }^{1}$ Grupo de investigación Espondiloartropatias,

Universidad de La Sabana-Hospital Militar Central, Chía, Colombia; ${ }^{2}$ Hospital

General de México, Ciudad de México, Mexico; ${ }^{3}$ Universidad del Norte,

Barranquilla; ${ }^{4}$ Asociación Colombiana de Reumatología, Bogotá, Colombia

Background: Within public health strategies, the quality of life is a fundamental factor of intervention. The EQ-5D-3L is one of the most used instruments worldwide.

Objectives: To describe the change in quality of life and functional limitation of patients with rheumatic diseases compared to a healthy population and patients with other systemic diseases.

Methods: The EQ-5D-3L survey was used in 6693 people from 6 cities in Colom bia. An analytical study was developed.

Results: In general, the healthy population $(n=1104)$ reported not to have problems according to the EQ-5D-3L (median: 98,34). Of the non-rheumatic patients ( $n=642), 20 \%$ showed a moderate compromise because of pain and discomfort, as well as anxiety and depression. Twenty percent of patients with cardiovascula

Abstract AB1244 - Table 1. Dimensions of EQ-5D-3L in patients with non-rheumatic diseases

\begin{tabular}{|c|c|c|c|c|c|c|c|c|c|c|}
\hline & & $\begin{array}{c}\text { HBP } \\
\mathrm{n}=201\end{array}$ & $\begin{array}{c}\text { Venous insufficiency } \\
\mathrm{n}=190\end{array}$ & $\begin{array}{c}\text { Migraine } \\
\mathrm{n}=158\end{array}$ & $\begin{array}{c}\text { Mental diseases } \\
\mathrm{n}=137\end{array}$ & $\begin{array}{c}\text { Obesity } \\
\mathrm{n}=95\end{array}$ & $\begin{array}{c}\text { Diabetes } \\
\mathrm{n}=55\end{array}$ & $\begin{array}{l}\text { CVD } \\
\mathrm{n}=53\end{array}$ & $\begin{array}{c}\text { Cancer } \\
n=13\end{array}$ & $\begin{array}{c}\text { Stroke } \\
\mathrm{n}=11\end{array}$ \\
\hline \multirow[t]{3}{*}{ MOBILITY } & No problems & 93,5 & 95,8 & 98,1 & 97,1 & 93,7 & 96,4 & 90,6 & 100 & 81,8 \\
\hline & $\begin{array}{l}\text { Some } \\
\text { problems }\end{array}$ & 6,5 & 4,2 & 1,9 & 2,9 & 6,3 & 3,6 & 9,4 & 0 & 18,2 \\
\hline & $\begin{array}{l}\text { Confined to } \\
\text { bed }\end{array}$ & 0 & 0 & 0 & 0 & 0 & 0 & 0 & 0 & 0 \\
\hline \multirow[t]{3}{*}{ SELF-CARE } & No & 97,5 & 97,9 & 98,7 & 100 & 98,9 & 98,2 & 94,3 & 100 & 90,9 \\
\hline & Moderate & 2 & 2,1 & 0,6 & 0 & 1,1 & 1,8 & 3,8 & 0 & 9,1 \\
\hline & Extreme & 0,5 & 0 & 0,6 & 0 & 0 & 0 & 1,9 & 0 & 0 \\
\hline USUAL & No problems & 95,5 & 97,4 & 99,4 & 99,3 & 97,9 & 100 & 92,5 & 100 & 90,9 \\
\hline \multirow[t]{2}{*}{ ACTIVITIES } & $\begin{array}{l}\text { Some } \\
\text { problems }\end{array}$ & 4 & 2,6 & 0,6 & 0,7 & 2,1 & 0 & 7,5 & 0 & 9,1 \\
\hline & Unable to & 0,5 & 0 & 0 & 0 & 0 & 0 & 0 & 0 & 0 \\
\hline PAIN/ & No & 87,1 & 89,5 & 86,1 & 84,7 & 88,4 & 92,7 & 84,9 & 92,3 & 81,8 \\
\hline \multirow[t]{2}{*}{ DISCOMFORT } & Moderate & 11,9 & 9,5 & 12 & 14,6 & 11,6 & 5,5 & 13,2 & 7,7 & 18,2 \\
\hline & Extreme & 1 & 1,1 & 1,9 & 0,7 & 0 & 1,8 & 1,9 & 0 & 0 \\
\hline ANXIETY/ & No & 91 & 93,7 & 92,4 & 76,6 & 94,7 & 96,4 & 84,9 & 92,3 & 90,9 \\
\hline \multirow[t]{2}{*}{ DEPRESSION } & Moderate & 8,5 & 6,3 & 7,6 & 22,6 & 5,3 & 1,8 & 15,1 & 7,7 & 9,1 \\
\hline & Extreme & 0,5 & 0 & 0 & 0,7 & 0 & 1,8 & 0 & 0 & 0 \\
\hline
\end{tabular}


disease $(n=53)$ reported moderate limitation for mobility. Patients with cardiovascular diseases reported moderate difficulties in carrying out daily activities.

* The values of each of the dimensions of the instrument are expressed in\%

** HBP: high blood pressure, CVD: cardiovascular disease, TB: tuberculosis

*** Mental disorders: anxiety, nervousness or depression.

Patients with rheumatic diseases $(n=2274)$ manifested different degrees of limitation in mobility and daily activities. Patients with rheumatic diseases and comorbidities had greater compromise in quality of life predominately by pain and physical discomfort (more than $50 \%$ of the patients) figure 1.

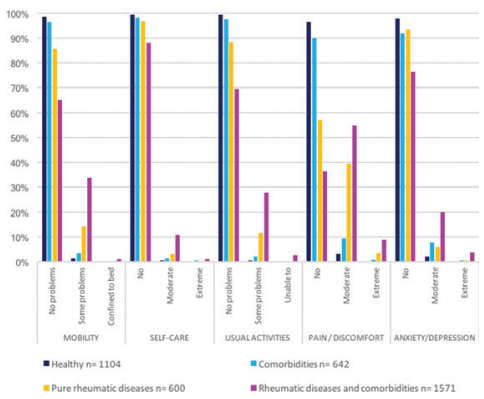

Abstract AB1244 - Figure 1. EQ5D-3L in patients with comorbidities, rheumatic diseases and healthy patients

Conclusions: In comparison with general population, rheumatic patients had a lower quality of life, and it is even worse in patients with rheumatic diseases and comorbidities. Comprehensive care of rheumatic patients should include strategies to improve standards of quality of life such as mobility, to perform daily activities and management of problems such as pain and discomfort. There are specific factors of intervention to reduce long-term disability of patients with rheumatic diseases.

Disclosure of Interest: None declared

DOI: 10.1136/annrheumdis-2018-eular.4319

\section{AB1245 $\quad$ EFFECTS OF A WORKPLACE-CENTRED COUNSELLING OF INDIVIDUALS WITH MUSCULOSKELETAL COMPLAINTS: A PROSPECTIVE COHORT STUDY}

H. Leiss, M. Hucke, M. Bécède, J. Smolen, K. Machold. Department of Rheumatology, Medical University of Vienna, Vienna, Austria

Background: Actively employed people with musculoskeletal complaints frequently seek medical advice only when symptoms have become chronic and have led to loss of workability.

Objectives: In this study, a brief examination was offered in the workplace setting in order to detect and to counsel individuals with symptoms of Rheumatic and musculoskeletal diseases(RMDs).

Methods: Employees of four companies were sent a screening questionnaire regarding musculoskeletal problems. In case of a positive screening, consultation by RMD specialists was offered which took place close to the workplace. If necessary, participants were referred to a practice/clinic specialised in RMDs (Orthopaedics, Rheumatology, Physical Medicine). Employees' work was categorised into physically highly demanding(HD) and less demanding(LD).

From participants consenting to follow-up, additional data were acquired: demographics, known pre-existing RMD, pain intensity, affected region(s), current treatment, number of sick leave-days due to musculoskeletal complaints, and out of pocket costs for treatments during the preceding year. General wellbeing and depression were measured by Euroquol-5d(EQ-5d) and Hospital Anxiety and Depression Scale(HADS). After one year, information about general wellbeing, pain intensity, treatment, individual costs, and days of sick leave during the intervening year was collected by telephone-interview.

Results: 6170 employees were invited.413 participated in the counselling program, 344 were enrolled in the study. $56.6 \%$ of the participants had no previously diagnosed RMD, after the specialists' assessment, this percentage decreased to $35.7 \%$. Men with LD workload had significantly higher wellbeing(EQ-5d scale):77.3 \pm 15.1 compared to women with both $\operatorname{LD}(71.0 \pm 20.1, p=0.034)$ and $H D$ $(64.6 \pm 21.3, p=0.001) . L D$ and HD differed significantly regarding percentage with painful upper(28.6 vs. $45.3, p=0.006)$ and lower(49.6 vs. $65.3, p=0.016)$ limbs.
Back pain was distributed equally among all groups. HD women reported significantly higher use of NSAIDs $(55.1 \%$ vs. $27.7 \%$ in female LD, $21.7 \%$ in male $\mathrm{HD}, 23.5 \%$ in male LD, $\mathrm{p}=0.001)$. HD men showed the lowest (4.1 \pm 2.9$)$, HD women showed the highest HADS anxiety-score $(6.3 \pm 3.8, \mathrm{p}=0.042)$.

235 individuals participated in telephone follow-up. There was significant improve ment in wellbeing(mean $77.2 \pm 17.4$ vs. $73.6 \pm 18.2$ at baseline, $p=0.006$ ) and in rating of RMD pain(mean $27.8 \pm 24.9$ vs. $40.8 \pm 24.6$ at baseline, $p=0.001$ ) Participants who were suspected by the specialist to suffer from RMDs had significantly increased out of pocket costs after one year(mean in $€ 441.8 \pm 861.6$ vs. $254.1 \pm 407.0, p=0.026)$. Use of NSAIDs decreased significantly from $29.1 \%$ to $17.4 \%, p=0.02$. Converesely, rates of use of physiotherapy (7.6 vs. $24.7, p=0.001$ ), gymnastics(2.7 vs. 23.4, $\mathrm{p}=0.001)$, physical therapy(12.8 vs. 43.3, $\mathrm{p}=0.027)$ and complementary/alternative methods $(7.4$ vs. $13.2, p=0.003)$ were significantly increased.

Conclusions: In our study we found most physical and psychological problems related to RMDs in HD working women. After one year, participants reported improved quality of life, reduction of RMD pain, higher utilisation of medical services and of gymnastics, less use of NSAIDs, and, if suspected to suffer from RMDs, higher out of pocket costs. Thus, this brief workplace-centred intervention appears to have had beneficial effects on both subjective well-being and on objective parameters suggesting improvements in physical/physiological health.

Disclosure of Interest: None declared

DOI: 10.1136/annrheumdis-2018-eular.4933

\section{AB1246 PROVISION OF RHEUMATOLOGY SERVICES TO 30 MILLION PEOPLE IN NORTH-WESTERN PAKISTAN (A NAÏVE DEPARTMENT WITH HUGE CHALLENGES)}

S. Khan ${ }^{1}$, I. Malik ${ }^{1}$, Z. Afridi ${ }^{2} .{ }^{1}$ Rheumatology; ${ }^{2}$ Pediatrics, Lady Reading Hospital, Medical Teaching Institution, Peshawar, Pakistan

Background: Practicing rheumatology needs multidisciplinary team work and also good funding. This becomes difficult in places where proper structure of healthcare is lacking.

Objectives: To share experience of establishing a Rheumatology unit in Khyber Pukhtunkhwa (KP) Pakistan.

Methods: KP is the Northwestern province of Pakistan. The population of KP is 30 million. The per capita income of Pakistan was 1180 US \$ in 2016.

Modern day treatments are expensive in rheumatology. Difficulties are in areas of expertise and biologics.

The first ever Rheumatology unit was established in Lady Reading Hospital (LRH) Peshawar in July 2017 which started its regular outpatient services. Problems at the start were absence of specialist nurses, junior doctors, special immunology and MSK Radiologists. Regular MSK ultrasound was started along with routine procedures. This had an enormous impact on the quality of care. Lack of awareness about rheumatic diseases in general population has been an issue which was addressed through newspapers, television and social media. The response was excellent. The outpatient numbers have raised, referral pathway was established and more patients are now seen in outpatients. Team was further built up by acquiring a trainee registrars and a consultant rheumatologist. Another problem was lack of proper patients education system due to lake of specialist nurses and non- availability of literature in local languages. Biologics are costly and very few people can afford these. Pakistan Bailtul Maal, a charitable organisation is the only way to provide biologics to patients on need basis. Currently only few biolog ics are available in the market i.e Etanercept, Rituximab and Tocilizumab. Adalimumab will come to market sometime in 2018.

Kids with Juvenile Idiopathic arthritis, Systemic Lupus Erythematosis and othe rheumatic problems are difficult to manage as there is no Paediatric Rheumatologist available in the entire province. We now have established a rapport with our paediatric colleagues which is working well.

Pakistan has only seven hospitals where training is offered in rheumatology but all are based in other provinces and none in KP. For this purpose we are in the process of establishing a dedicated rheumatology department where full training will be given to trainees according to international standards.

The data on rheumatic diseases is non-existing so we are now working on data collection on our local population.

Results: Working as a rheumatologist is a big challenge in under resourced areas. We have been having worst case scenario in almost all aspects. Howeve someone has to be at the forefront as millions of people have rheumatic diseases and they cannot be left alone with these conditions untreated. 